\title{
Detrimental effects of lipopolysaccharides on maturation of bovine oocytes
}

\author{
Shanjiang Zhao ${ }^{1, a}$, Yunwei Pang ${ }^{1, a}$, Xueming Zhao ${ }^{1}$, Weihua Du', Haisheng Hao ${ }^{1}$, and Huabin Zhu ${ }^{1, *}$
}

* Corresponding Author: Huabin Zhu Tel: +86-010-62895971, Fax: +86-010-62895971,

E-mail: zhuhuabin@caas.cn

1 Embryo Biotechnology and Reproduction Laboratory, Institute of Animal Science, Chinese Academy of

Agricultural Sciences, Beijing 100193, China

a Shanjiang Zhao and Yunwei Pang contributed equally to this work.

\section{ORCID}

Shanjiang Zhao

https://orcid.org/0000-0001-5324-9182

Yunwei Pang

https://orcid.org/0000-0002-7312-179X

Xueming Zhao

https://orcid.org/0000-0001-6110-2690

Weihua Du

https://orcid.org/0000-0003-1248-4471

Haisheng HaO

https://orcid.org/0000-0002-8232-7909

Huabin Zhu

https://orcid.org/0000-0001-7970-9183

Submitted Jul 20, 2018; Revised Sept 13, 2018; Accepted Oct 16, 2018
Objective: Gram-negative bacteria lipopolysaccharide (LPS) has been reported to be associated with uterine impairment, embryonic resorption, ovarian dysfunction, and follicle retardation. Here, we aimed to investigate the toxic effects of LPS on the maturation ability and parthenogenetic developmental competence of bovine oocytes.

Methods: First, we developed an in vitro model to study the response of bovine cumulusoocyte complexes (COCs) to LPS stress. After incubating germinal vesicle COCs in $10 \mu \mathrm{g} / \mathrm{mL}$ of LPS, we analyzed the following three aspects: the expression levels of the LPS receptor tolllike receptor 4 (TLR4) in COCs, activities of intracellular signaling protein $\mathrm{p} 38$ mitogen-activated protein kinase ( $\mathrm{p} 38 \mathrm{MAPK})$ and nuclear factor-kappa B (NF- $\mathrm{kB}$ ); and the concentrations of interleukin (IL)-1 $\beta$, tumor necrosis factor (TNF)- $\alpha$, and IL-6. Furthermore, we determined the effects of LPS on the maturation ability and parthenogenetic developmental competence of bovine oocytes.

Results: The results revealed that LPS treatment significantly elevated TLR4 mRNA and protein expression levels in COCs. Exposure of COCs to LPS also resulted in a marked increase in activity of the intracellular signaling protein p-p38 MAPK and NF- $k B$. Furthermore, oocytes cultured in maturation medium containing LPS had significantly higher concentrations of the proinflammatory cytokines IL-1 $\beta$, TNF- $\alpha$, and IL- 6 . LPS exposure significantly decreased the first polar body extrusion rate. The cytoplasmic maturation, characterized by polar body extrusion and distribution of peripheral cortical granules, was significantly impaired in LPStreated oocytes. Moreover, LPS exposure significantly increased intracellular reactive oxygen species levels and the relative mRNA abundance of the antioxidants thioredoxin (Trx), $\operatorname{Tr} x 2$, and peroxiredoxin 1 in oocytes. Moreover, the early apoptotic rate and the release of cytochrome $C$ were significantly increased in response to LPS. The cleavage, morula, and blastocyst formation rates were significantly lower in parthenogenetically activated oocytes exposed to LPS, while the incidence of apoptotic nuclei in blastocysts was significantly increased.

Conclusion: Together, these results provide an underlying mechanism by which LPS impairs maturation potential in bovine oocytes.

Keywords: Cattle; Lipopolysaccharide; Oocyte; Oxidative Stress; Apoptosis

\section{INTRODUCTION}

Lipopolysaccharide (LPS) is a toxic component occurring in the cell walls of gram-negative bacteria and has been found to cause delayed ovulation and reduced fecundity in cattle $[1,2]$. In general, bacterial infections of the uterus are ubiquitous in human and animals, including cattle, after coitus and/or parturition [3], and up to $20 \%$ of animals failed to achieve pregnancy after infection of the endometrium with Escherichia coli [4,5]. Many studies have reported that LPS accumulation in the follicular fluid of animals with metritis may cause ovarian dysfunction, aberrant changes in the oviductal transcriptome profile, and perturbation of follicular development [6-8]. 
LPS is known to initiate several destructive processes via interaction with toll-like receptor 4 (TLR4), which is a member of the TLR family of transmembrane proteins $[9,10]$. TLR4 binds to LPS, triggering a cascade of signal transduction events that evoke the release of numerous biochemical mediators such as cytokines and toxic free radicals $[8,11]$. Blum et al [12] studied dairy cows and found that LPS could induce tumor necrosis factor (TNF)- $\alpha$ and Nox (nitrite and nitrate) accumulation in milk and plasma. Bovine and murine granulosa cells express TLR4 receptor complex, respond rapidly to LPS with phosphorylation of the TLR signaling components p38 and extracellular signal-regulated kinase (ERK), and increase the abundance of interleukin (IL) 6 and IL8 transcripts [3,13]. In addition, LPS has been reported to directly affect the intracellular redox status and induce apoptotic incidence by enhancing the release of pro-apoptotic factors [14]. Thus, we hypothesize that LPS-induced immune challenges in bovine cumulus-oocyte complexes (COCs) have a deleterious effect on both the maturation and developmental competence of follicle-enclosed oocytes.

Mammalian oocyte maturation involves nuclear and cytoplasmic maturation, which are essential for successful fertilization and embryonic development [15]. The developmental competence of oocytes depends on follicular development and includes a variety of molecular and cellular events [16]. Higher and moderate LPS levels have been detected in the follicular fluid of growing follicles collected from postpartum cows with endometritis as well as from women with bacterial vaginosis $[3,17,18]$. Ibrahim et al $[8]$ found that the viability of oviduct epithelial cells was significantly decreased following LPS challenge. In the presence of LPS, oocytes failed to reach metaphase II (MII) or exhibited aberrant meiotic structures. However, the exact mechanisms by which LPS affects oocyte competence during in vitro maturation (IVM) remains unclear. Thus, in the present study we aimed to assess the detrimental effect of LPS on oocyte maturation potential, and to investigate the mechanisms involved in this process.

\section{MATERIALS AND METHODS}

\section{Antibodies and chemicals}

Unless otherwise specified, all chemicals were purchased from Sigma-Aldrich Co. (St. Louis, MO, USA). Rabbit polyclonal antibodies to nuclear factor-kappa B (NF-kB) and TLR4 were from Novus (Novus, Littleton, CO, USA). Rabbit polyclonal antibodies to cytochrome $C$ were obtained from Abcam (Cambridge, UK). p-p38 mitogen-activated protein kinase (p38 MAPK) and p-p38 MAPK were purchased from Cell Signaling Technology (Beverly, MA, USA). Alexa Fluor-488-conjugated mouse anti-rabbit secondary antibodies were purchased from Invitrogen (Carlsbad, CA, USA). Annexin V-FITC kits were purchased from Vazyme Biotech Co., Ltd. (Nanjing, China).
In Situ Cell Death Detection Kit was obtained from Roche (Mannheim, Germany). All experimental procedures were conducted according to the guidelines of the Institutional Animal Care and Use Committee at the Chinese Academy of Agricultural Sciences.

\section{Oocyte harvest and in vitro maturation}

Bovine ovaries were transported from the local abattoirs to the laboratory in sterile physiological saline solution, and maintained at $26^{\circ} \mathrm{C}$ to $30^{\circ} \mathrm{C}$ within $2 \mathrm{~h}$ of harvesting. Before ovaries were aspirated, they were washed three times with warm physiological saline. The COCs that were 2 to $6 \mathrm{~mm}$ in diameter were manually aspirated from antral follicles using an 18-gauge needle. Oocytes surrounded by at least three compact cumulus layers and had uniform ooplasm were selected for the next step. Generally, prior to IVM, COCs should be washed thrice in IVM medium. Then, 50 COCs were incubated in $750 \mu \mathrm{L}$ of maturation medium in each well of a four-well plate (Nunc, Denmark) in air containing $5 \% \mathrm{CO}_{2}$ at $38.5^{\circ} \mathrm{C}$ and maximum humidity. The IVM was carried out in TCM-199 (Gibco BRL, Grand Island, NY, USA) containing $0.01 \mathrm{IU} / \mathrm{mL}$ follicle-stimulating hormone, $10 \mathrm{IU} / \mathrm{mL}$ luteinizing hormone, $1 \mu \mathrm{g} / \mathrm{mL}$ estradiol, $10 \mathrm{mg} / \mathrm{mL}$ heparin, and $10 \%(\mathrm{v} / \mathrm{v})$ fetal bovine serum (FBS; Gibco BRL, USA).

\section{Lipopolysaccharide treatment}

LPS was dissolved in water and diluted with IVM medium to obtain a final concentrations of $10 \mu \mathrm{g} / \mathrm{mL}$. The proportion of LPS in the final culture medium should not exceed $1 \%$ after dilution with IVM medium.

\section{Parthenogenetic activation and in vitro culture}

Oocytes were harvested from bovine ovaries and pretreated with $10 \mu \mathrm{g} / \mathrm{mL}$ LPS for $22 \mathrm{~h}$. They were then subjected to parthenogenetic activation and in vitro embryo culture in the absence of LPS until they reached the blastocyst stage. Oocytes were treated with $5 \mu \mathrm{M} \mathrm{Ca}^{2+}$ ionophore A23187 for $5 \mathrm{~min}$ and incubated in $2 \mathrm{mM}$ of 6-dimethylaminopurine for another $6 \mathrm{~h}$. Following activation, oocytes were cultured in Charles Rosenkrans medium with added amino acid (CR1aa) supplemented with $0.1 \%(\mathrm{w} / \mathrm{v})$ bovine serum albumin (BSA) for $48 \mathrm{~h}$ at $38.5^{\circ} \mathrm{C}$ in air containing $5 \% \mathrm{CO}_{2}$. They were then cultured for an additional 5 days in CR1aa-supplemented medium with $10 \%(\mathrm{v} / \mathrm{v})$ FBS. The medium was changed every two days throughout the culture period. Cleavage, morula, and blastocyst formation rates were recorded at 2, 6, and 7 days, respectively. Each test was repeated three times, and 30 oocytes were analyzed per group in each replicate.

\section{Cell counting}

For cell counting, blastocysts were collected on day 7 and stained as previously described [19]. All blastocysts were washed with 
phosphate-buffered saline (PBS) containing $1 \mathrm{mg} / \mathrm{mL}$ polyvinyl alcohol (PVA), and fixed in $500 \mu \mathrm{L}$ ethanol containing $10 \mu \mathrm{g} / \mathrm{mL}$ Hoechst-33342 for $30 \mathrm{~min}$. After rinsing in $0.1 \%$ PVA/PBS, the stained blastocysts were mounted onto slides and analyzed under a confocal microscope (Olympus FV1000, Tokyo, Japan). Each test was repeated three times, and 30 oocytes were analyzed per group in each replicate.

\section{Cytochemical staining}

The COCs were cultured for $22 \mathrm{~h}$, after which cumulus cells were removed by repeated pipetting. Denuded oocytes were fixed in $3.7 \%(\mathrm{w} / \mathrm{v})$ paraformaldehyde in PBS for $1 \mathrm{~h}$, and permeabilized with PBS supplemented with $1 \%$ Triton X-100 for $1 \mathrm{~h}$. After the cells were washed three times in PBS containing $0.1 \%$ PVA, the oocytes were blocked in 1\% BSA/PBS for $8 \mathrm{~h}$. The oocytes were incubated with antibodies against TLR4 (1:500), NF- $\kappa B(1: 500)$, and cytochrome $C(1: 1,000)$ overnight at $4^{\circ} \mathrm{C}$. After washing three times ( 5 min each time) in PBS$0.1 \%$ PVA medium, the oocytes were labeled with secondary antibodies for $40 \mathrm{~min}$ at room temperature and then incubated with $2 \mu \mathrm{g} / \mathrm{mL}$ 4,6-diamidino-2-phenylindole (Roche, Germany). Samples from each group were transferred onto identical slides and analyzed under a confocal microscope (Olympus FV1000, Japan).

To study the distribution of peripheral cortical granules (CGs) and to determine the cellular levels of reactive oxygen species (ROS), the samples were stained with $10 \mu \mathrm{g} / \mathrm{mL}$ of fluorescein isothiocyanate (FITC)-labeled peanut agglutinin and $10 \mu \mathrm{M} 2^{\prime}, 7^{\prime}$-dichlorodihydrofluorescein diacetate in the dark for $30 \mathrm{~min}$, and then washed thrice in washing buffer. Then, apoptosis was detected using an In Situ Cell Death Detection Kit and an Annexin V/PI-FITC kit. Samples from each group were transferred onto identical slides and analyzed under a confocal microscope (Olympus FV1000, Japan).

Each test was repeated three times, and at least 15 oocytes were analyzed per group in each replicate. The fluorescence intensity was analyzed using ImageJ software (National Institutes of Health, Bethesda, MD, USA), and the fluorescence intensity per pixel within the images was analyzed using the ROI (National Institutes of Health, USA). Finally, the average values of all images were calculated and used as the final intensities for each group.

\section{Enzyme-linked immunosorbent assay}

IL- $1 \beta$, IL- 6 , and TNF- $\alpha$ were measured from cell-free supernatants using commercially available bovine-specific enzymelinked immunosorbent assay kits (LBTR, Beijing, China) according to the manufacturer's instructions. Each test was repeated three times.

\section{Real-time quantitative polymerase chain reaction}

The total RNA was extracted from 100 oocytes with or without LPS treatment using Trizol reagent (Invitrogen, USA) for 22 h. First-strand cDNA was synthesized from $0.05 \mu \mathrm{g}$ of RNA using random hexamers. Quantitative real-time polymerase chain reaction was conducted in three replicates using an ABI7900 SDS instrument (Applied Biosystems, Foster city, CA, USA), and the primers used are listed in Table 1 . The expression level of the genes was calculated using the $2^{-\Delta \Delta c(t)}$ method, and glyceraldehyde-3-phosphate dehydrogenase was used as the reference gene. Each test was repeated three times, and 100 oocytes were analyzed per group in each replicate.

\section{Western blot analysis}

For the Western blot analysis, 150 bovine oocytes at the MII stage were harvested and washed thrice in PBS. The proteins were extracted and seperated by sodium dodecyl sulfate-polyacrylamide gel electrophoresis using 7.5\% polyacrylamide gel and tranferred onto membranes (Millipore Corp, Billerica, MA, USA). The membranes were then blocked in $5 \%(\mathrm{w} / \mathrm{v})$ skim milk overnight at $4^{\circ} \mathrm{C}$, and incubated with p-p38 MAPK monoclonal antibodies (1:1,000 dilution) and p38 MAPK anti-

Table 1. Primer sequences used for real-time quantitative polymerase chain reaction

\begin{tabular}{|c|c|c|c|c|c|}
\hline Gene & Gene ID & Primer & Sequence $\left(5^{\prime}-3^{\prime}\right)$ & Annealing temperature $\left({ }^{\circ} \mathrm{C}\right)$ & Fragment size $(b p)$ \\
\hline \multirow[t]{2}{*}{ GAPDH } & 281181 & Forward & GGGTCATCATCTCTGCACCT & 60 & 177 \\
\hline & & Reverse & GGTCATAAGTCCCTCCACGA & & \\
\hline \multirow[t]{2}{*}{$T L R 4$} & 281536 & Forward & CTTGCGTACAGGTTGTTCCTAA & 56 & 153 \\
\hline & & Reverse & CTGGGAAGCTGGAGAAGTTATG & & \\
\hline \multirow[t]{2}{*}{$\operatorname{Tr} x$} & 280950 & Forward & TGGTGTTCCTTGAAGTAGATGTGG & 60 & 109 \\
\hline & & Reverse & TCACCCACCTTCTGTCCCTTT & & \\
\hline \multirow[t]{2}{*}{$\operatorname{Tr} \times 2$} & 281557 & Forward & GTTCCTGACCTCCATAATCTCCG & 61 & 121 \\
\hline & & Reverse & GGCTTGGCTGGGTGTTACTGT & & \\
\hline \multirow[t]{2}{*}{ Prx1 } & 281997 & Forward & CGCTGGTGTCGGTCCTATTT & 60 & 98 \\
\hline & & Reverse & GCTGTTGCTTTGAACTGGGG & & \\
\hline \multirow[t]{2}{*}{ Grx } & 515416 & Forward & TACCTCGGGTCTTCATCGGT & 55 & 103 \\
\hline & & Reverse & GCTCCCATTTGCTTTAGCCG & & \\
\hline
\end{tabular}

GAPDH, glyceraldehyde-3-phosphate dehydrogenase; TLR4, toll-like receptor 4; Trx, thioredoxin; Prx1, peroxiredoxin 1; Grx, glutaredoxin. 
bodies $(1: 1,000)$ at $4^{\circ} \mathrm{C}$ overnight, washed thrice in TBS for five minutes each time, and incubated with horseradish peroxidaseconjugated secondary antibodies (Zhong Shan Biotechnology, Beijing, China) for $1 \mathrm{~h}$ at room temperature. The signals were detected using an ECL kit (Tanon, Shanghai, China). Each test was repeated three times, and 150 oocytes were analyzed per group in each replicate.

\section{Statistical analysis}

At least three biological replicates were used, and the results were presented as means \pm stsandard error of the mean. Data were statistically analyzed using the $t$ test. Less than $0.05 \mathrm{p}$ value was considered statistically significant.

\section{RESULTS}

Lipopolysaccharide exposure induces inflammation in bovine cumulus-oocyte complexes

We first measured the expression of TLR4 in bovine COCs. The TLR4 is one of the pattern recognition receptors for LPS, which activates the downstream factors p38 MAPK and NF- $k B$.
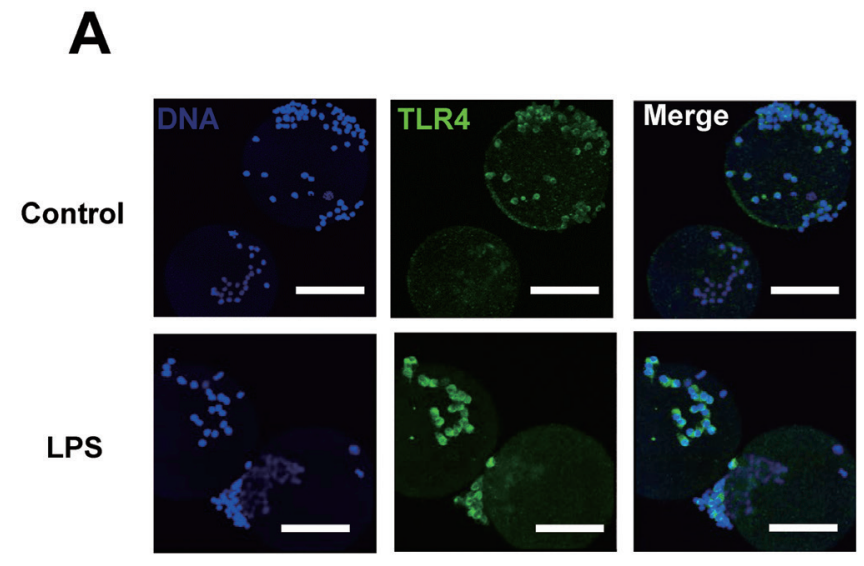

C
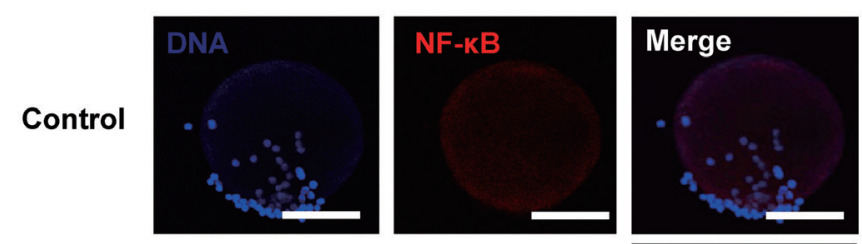

LPS
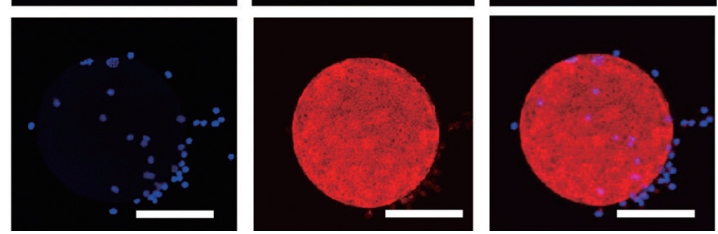

Excitation of NF- $\kappa \mathrm{B}$ results in the generation of cytokines and other inflammatory mediators such as TNF- $\alpha$, IL-1 $\beta$, and IL-6. The TLR4 mRNA level was upregulated in the LPS group compared with the control group ( $\mathrm{p}<0.05$; Figure $2 \mathrm{~A}$ ), and TLR4 and NF- $\mathrm{KB}$ protein expression levels were also significantly increased in the LPS group relative to the control (Figure 1). We also measured the concentrations of three proinflammatory cytokines, IL- $1 \beta$, TNF- $\alpha$, and IL- 6 in cell-free supernatants, and the results revealed that IL- $1 \beta$, TNF- $\alpha$, and IL- 6 concentrations were significantly higher in the LPS group than in the control group $(\mathrm{p}<0.05$; Figure $2 \mathrm{~B})$.

To further confirm the effects of LPS on maturation potential, we analyzed the level of p38 MAPK, which is a typical inflammatory gene expression regulator. As shown in Figure $2 \mathrm{C}$ and 2D, the p-p38 MAPK level was significantly higher in the LPS-treated oocytes than in the control oocytes $(\mathrm{p}<0.05)$. Collectively, these results indicate that LPS challenge induces an inflammatory response in bovine COCs, which may in turn lead to the low maturation rate of oocytes.

Lipopolysaccharide exposure inhibits the maturation

B

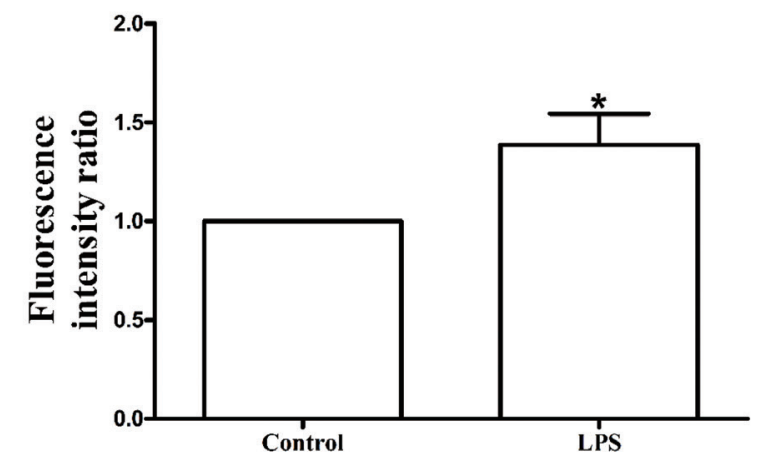

D

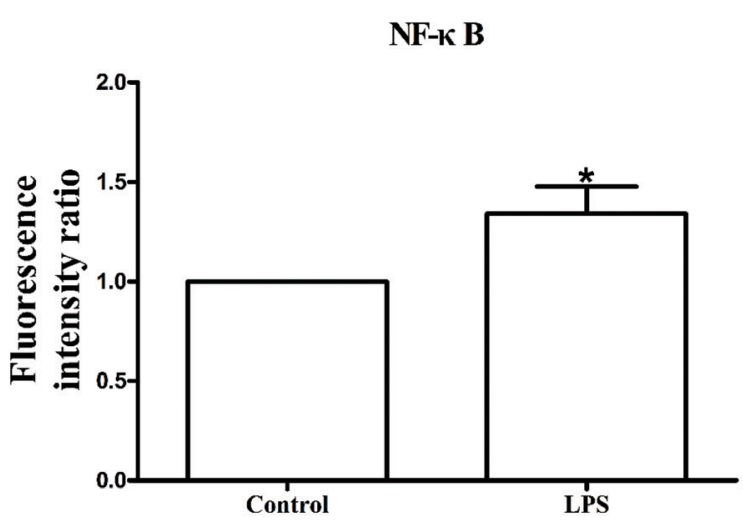

Figure 1. Lipopolysaccharide exposure increases the TLR4 and NF- $\kappa B$ levels in bovine cumulus-oocyte complexes (COCs). (A) Immunofluorescence staining for TLR4 in bovine COCs. Blue, chromatin; green, TLR4. Bar = $50 \mu \mathrm{m}$. (B) TLR4 levels in bovine COCs. (C) Immunofluorescence staining for NF- $\mathrm{kB}$ in bovine COCs. Blue, chromatin; red,

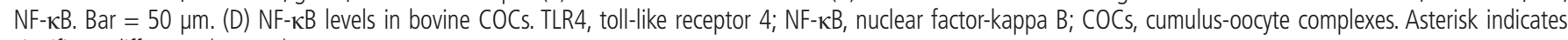
significant difference $(p<0.05)$. 

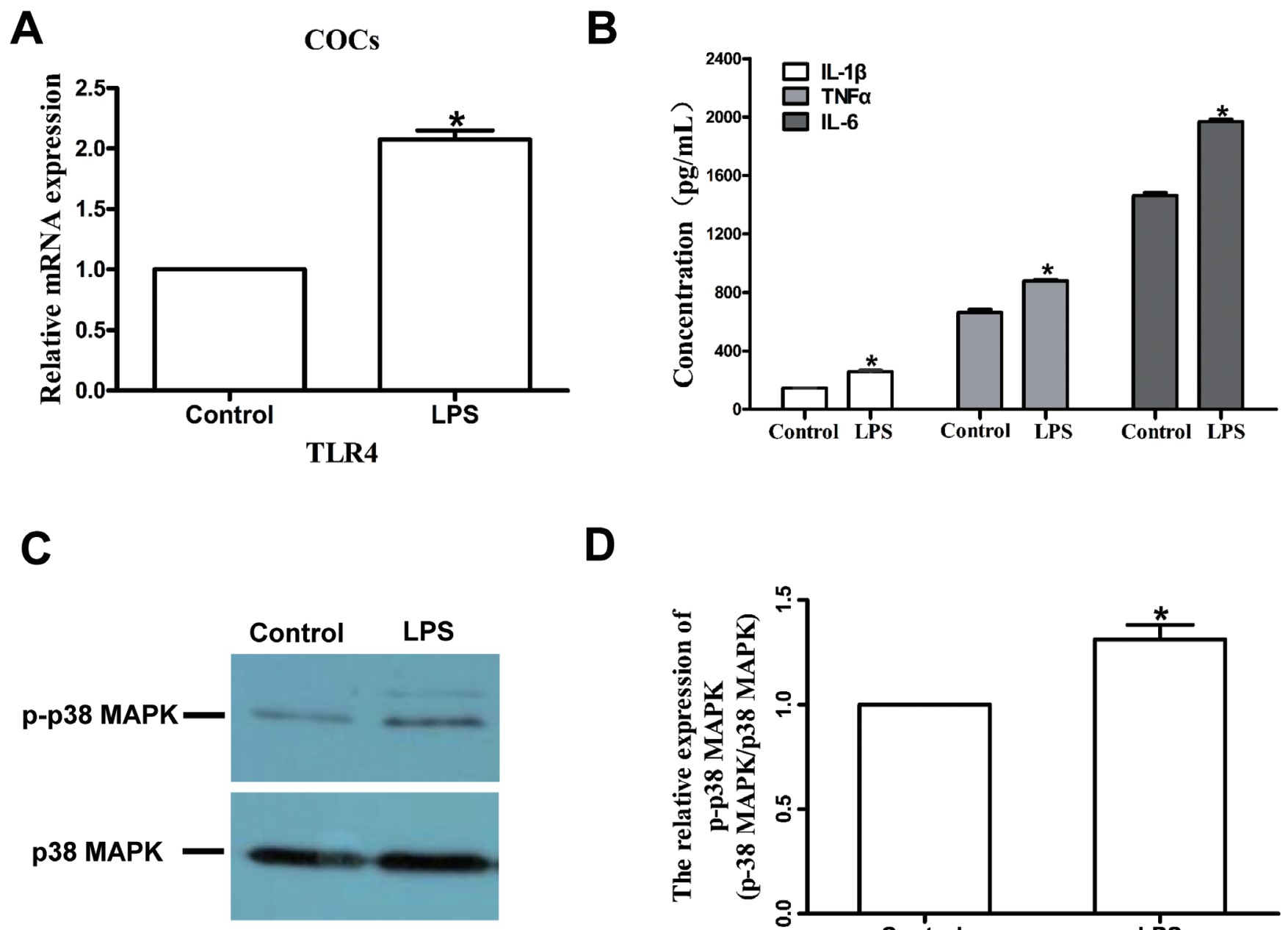

\section{D}

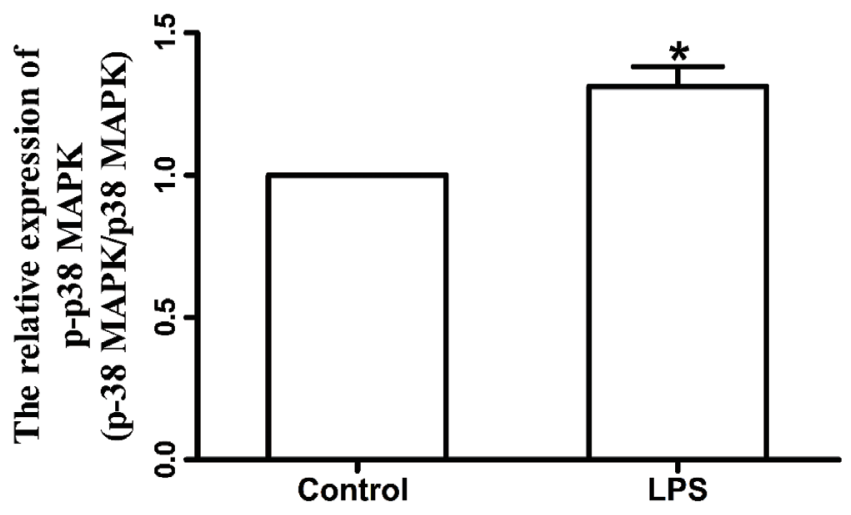

Figure 2. LPS exposure increases the TLR4 mRNA level, p-p38 MAPK protein level, and production of the proinflammatory cytokines IL-1 $\beta$, TNF- $\alpha$, and IL-6. (A) TLR4 transcript levels in bovine COCs. (B) Production of IL-1 $\beta$, TNF- $\alpha$, and IL- 6 after exposure to LPS. (C) and (D) Western blot analysis of p-p38 MAPK and p-38 protein levels. LPS, lipopolysaccharide; TLR4, toll-like receptor 4; p-p38 MAPK, phosphorylation of p38 mitogen-activated protein kinase; IL, interleukin; TNF- $\alpha$, tumor necrosis factor- $\alpha$; COCs, cumulus-0ocyte complexes. Asterisk indicates significant difference $(p<0.05)$.

potential of bovine oocytes

Exposing oocytes to LPS significantly decreased the first polar body extrusion rate $(64.8 \% \pm 3.89 \%, \mathrm{n}=402)$ compared with the control group $(86.5 \% \pm 1.36 \%, \mathrm{n}=386)(\mathrm{p}<0.05$; Figure $3 \mathrm{~A})$. The proportion of oocytes with peripheral CG distribution $(26.7 \% \pm 3.33 \%, \mathrm{n}=80)$ was also significantly lower in the LPStreated group than in the control group $(57.8 \% \pm 2.94 \%, \mathrm{n}=$ 80) $(\mathrm{p}<0.05$; Figure 3B, 3C). These results indicate that LPS exposure directly inhibits the maturation potential of bovine oocytes.

Lipopolysaccharide exposure induces oxidative stress and apoptosis in bovine oocytes

LPS treatment significantly increased the intracellular ROS levels in MII oocytes compared to the control group ( $\mathrm{p}<0.05$; Figure $4 \mathrm{~A}, 4 \mathrm{~B})$. The mRNA transcripts of thioredoxin (Trx), $\operatorname{Tr} x 2$, and peroxiredoxin 1 ( $\operatorname{Pr} x 1)$ were markedly decreased following LPS treatment, while glutaredoxin (Grx) mRNA level was unaffected ( $p>0.05$; Figure 4 C), illustrating that LPS treatment induces oxidative stress in bovine oocytes.

The results of the Annexin V assay revealed a significantly higher early apoptosis rate in oocytes exposed to LPS (26.33\% $\pm 2.33 \%, \mathrm{n}=72$ ) than in untreated oocytes in the control group $(12.67 \% \pm 1.45 \%, \mathrm{n}=66)(\mathrm{p}<0.05$; Figure 5A, 5B). Furthermore, the fluorescence signal of cytochrome $C$ was also significantly enhanced in the cytoplasm of LPS-treated oocytes $(n=80$; $p<$ 0.05 , Figure $5 \mathrm{C}, 5 \mathrm{D}$ ). These data suggest that the detrimental effect of LPS on oocyte maturation potential may be partly due to its pro-apoptotic activity.

Lipopolysaccharide exposure decreases parthenogenetic development of bovine oocytes We further analyzed the effects of LPS on the parthenogenetic development of bovine oocytes. The cleavage $(73.3 \% \pm 1.76 \%$, 


\section{A Polar body extrusion of COCs groups}

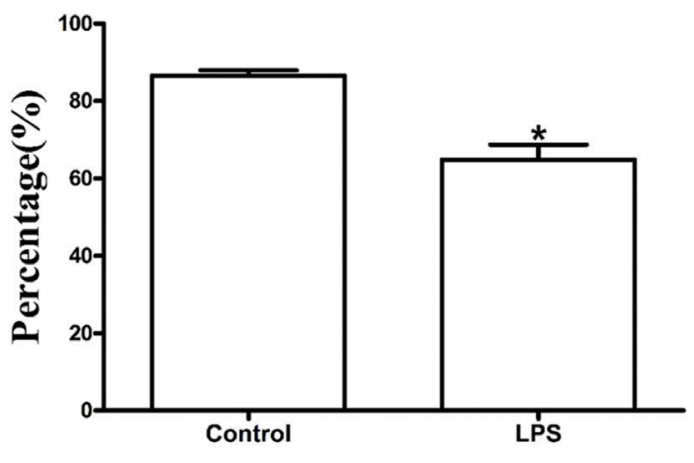

B
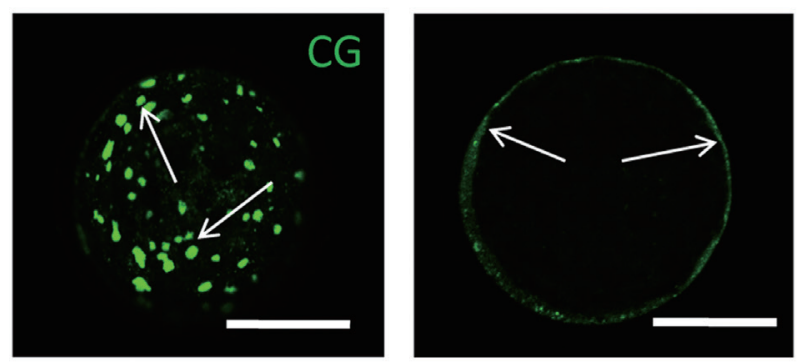

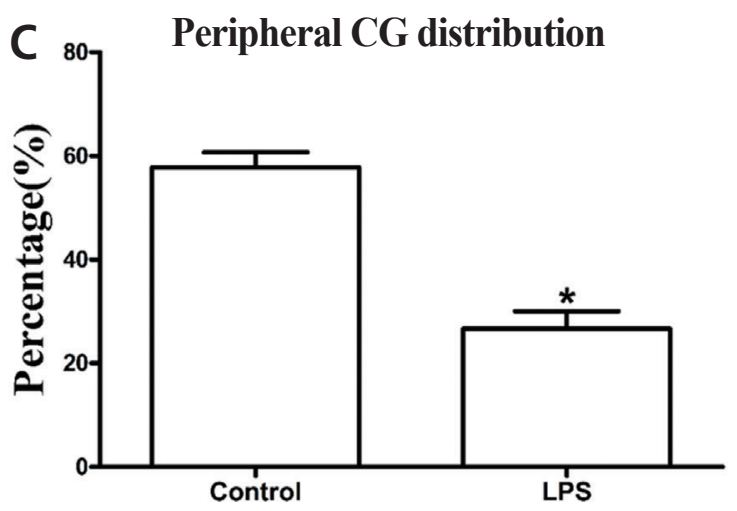

Figure 3. LPS exposure inhibits the maturation potential of bovine oocytes. (A) Polar body extrusion rate after LPS treatment. (B) Representative photomicrographs. Peripheral CGs are indicated with arrows. Green, CG. Bar $=50 \mu \mathrm{m}$. (C) Proportion of oocytes with peripheral CG distribution after LPS treatment. LPS, lipopolysaccharide; CGs, cortical granules. Asterisk indicates significant difference $(p<0.05)$.

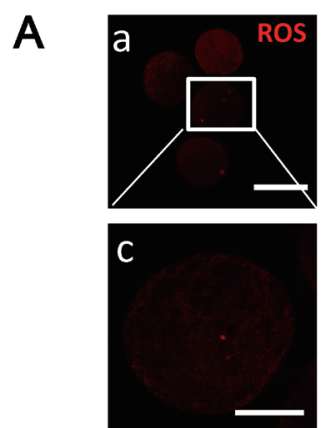

Control
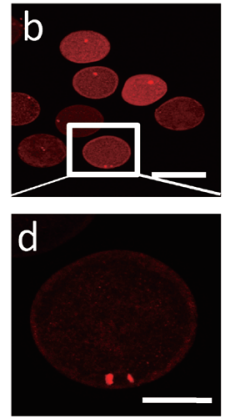

LPS
B

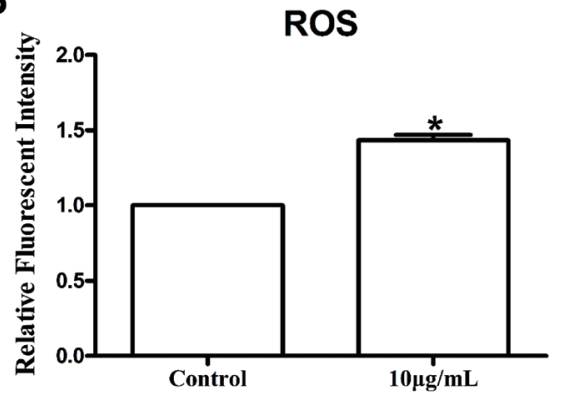

C

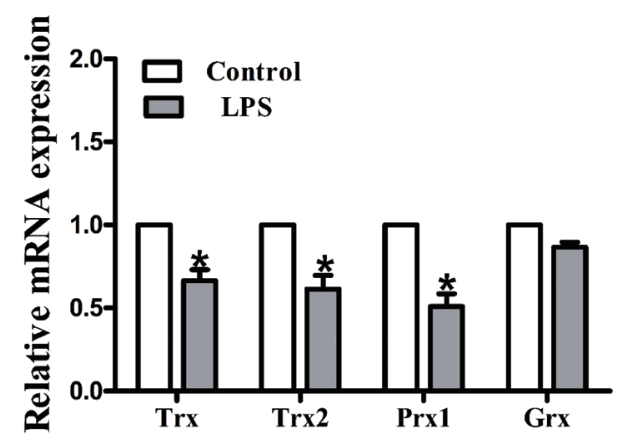

Figure 4. LPS treatment alters intracellular redox state in bovine oocytes. (A) Representative photomicrographs. Red, ROS. $a, b: B a r=100 \mu m ; c, d: B a r=50 \mu m$. (B) ROS expression after LPS treatment. (C) Transcripts of redox signaling-associated genes Trx, Trx2, Prx1, and Grx. LPS, lipopolysaccharide; ROS, reactive oxygen species; Trx, thioredoxin; Prx1, peroxiredoxin 1; Grx, glutaredoxin. Asterisk indicates significant difference $(p<0.05)$. 
A

Control

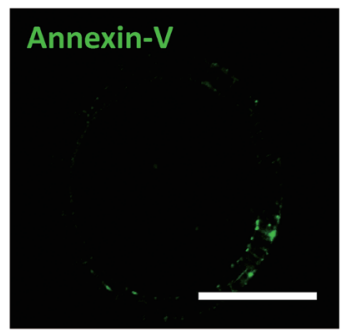

C

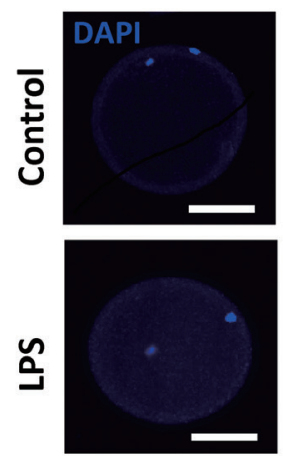

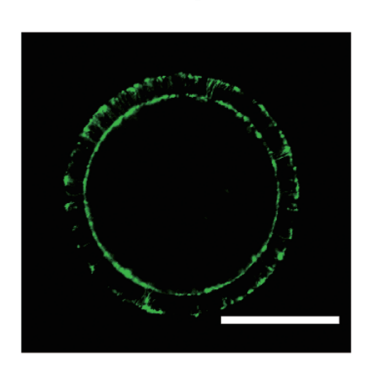

LPS
B

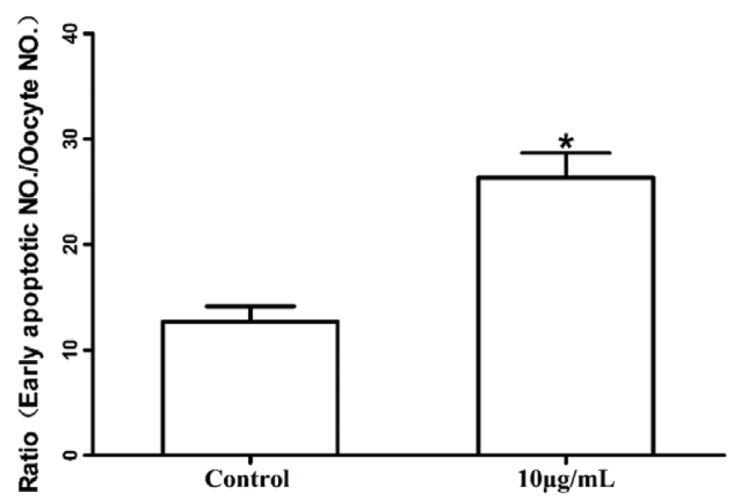

D

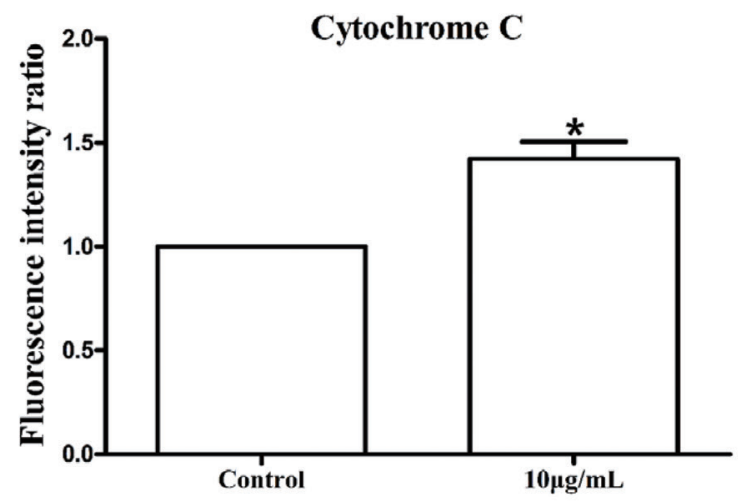

Figure 5. LPS exposure induces apoptosis in bovine oocytes. (A) Representative photomicrographs. Green, Annexin V. Bar $=50 \mu m$. (B) Rates of early apoptosis after LPS treatment. (C) Representative photomicrographs. Green, cytochrome C. Bar $=50 \mu \mathrm{m}$. (D) Cytochrome C levels in bovine oocytes. LPS, lipopolysaccharide. Asterisk indicates significant difference $(p<0.05)$.

$\mathrm{n}=230)$, morula $(30.7 \% \pm 0.88 \%)$, and blastocyst formation $(14.8 \% \pm 1.96 \%)$ rates were significantly lower in the LPS group than in the control group $(82.3 \% \pm 0.88 \%, \mathrm{n}=248 ; 47.3 \% \pm 2.40 \%$; and $34.2 \% \pm 0.8 \%$, respectively) ( $<<0.05$; Figure $6 \mathrm{~A}$ ). The average number of total cells in blastocysts did not significantly differ between the control $(89.3 \% \pm 1.75 \%, \mathrm{n}=30)$ and treatment $(85.2 \% \pm 2.05 \%, \mathrm{n}=30)$ groups ( $>0.05$; Figure $2 \mathrm{~B}$ ). However, LPS treatment significantly increased the incidence of apoptotic nuclei in parthenogenetic blastocysts $(3.33 \pm 0.20, \mathrm{n}=32)$ compared to the control group $(2.06 \pm 0.15, \mathrm{n}=32)(\mathrm{p}<0.05$; Figure $6 \mathrm{C}, 6 \mathrm{D})$. Together, these data imply that exposure of bovine oocytes to LPS during IVM affected their quality and reduced their ability to subsequently undergo parthenogenetic development.

\section{DISCUSSION}

We first developed an in vitro model of LPS stress in bovine COCs. The current study was conducted to test the effects of LPS on the maturation ability and parthenogenetic developmental competence of bovine oocytes. The results demonstrated that LPS exposure significantly decreased the maturation rate of bovine oocytes and induced oxidative stress and apoptosis during the maturation of bovine oocytes. In addition, the development and quality of bovine parthenotes produced in vitro and derived from LPS-treated oocytes were obviously impaired. During ovulation, COCs generate a physical matrix around the oocyte; in addition, cumulus and granulosa cells also express considerable immune cell-like function [20]. It is well established that TLRs, which can recognize pathogen-associated molecules such as LPS, play important roles in initiating inflammatory responses orchestrated by proinflammatory cytokines such as IL-1, IL-6, and TNF- $\alpha$ [21]. In cows, granulosa cells trigger an immune response to LPS via the TLR4 pathway, leading to inflammation and perturbation of meiotic competence [13]. COCs exposed to LPS exhibit reinforced phosphorylation levels of p38 MAPK, increased ERK1/2 and NF- $\kappa B$ activity, and increased IL- 6 and TNF- $\alpha$ expression levels. As phosphorylation of p38 MAPK and induction of IL- 6 and TNF- $\alpha$ expression are downstream events initiated by activation of the TLR pathway, Shimada et al [20] proposed that the TLR pathway may play critical roles during the ovu- 
A

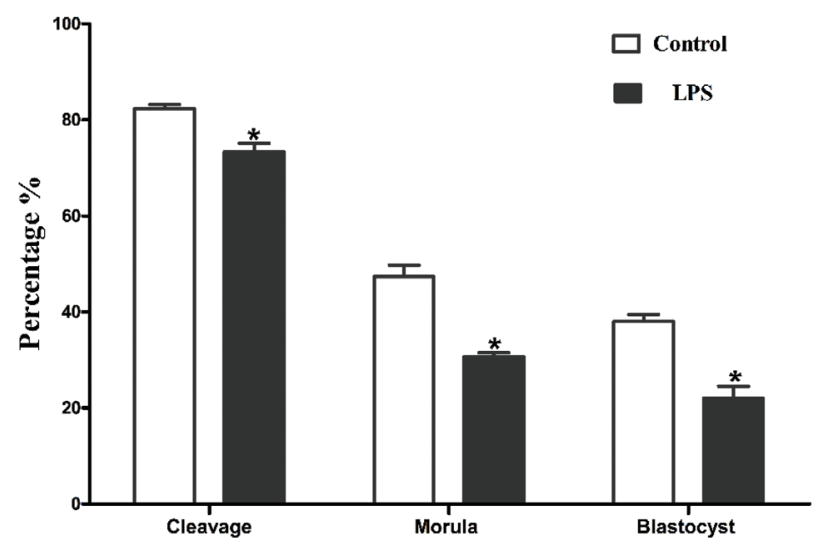

C
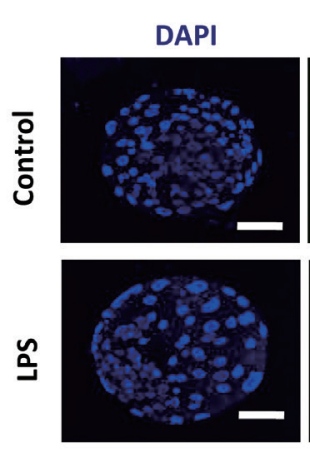
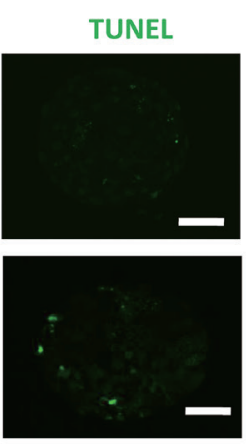

B

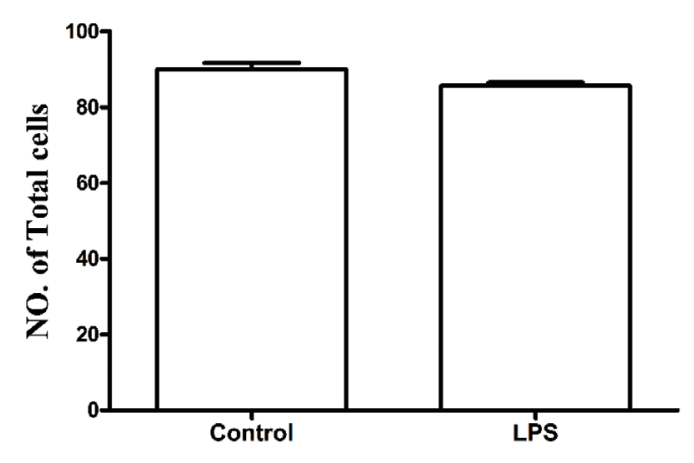

D

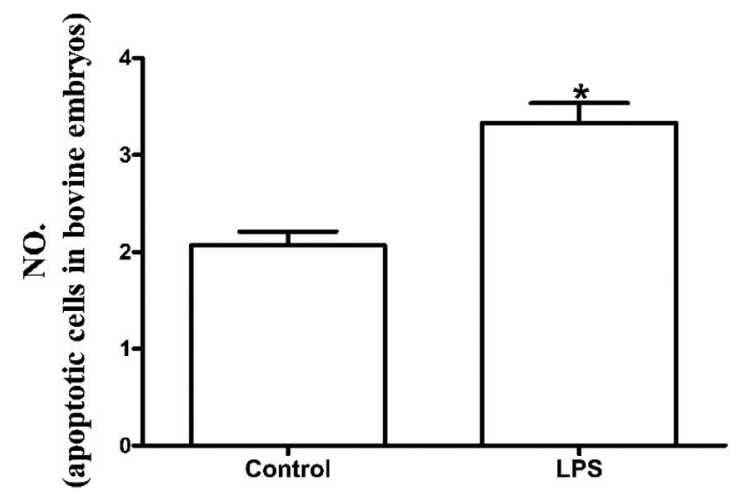

Figure 6. LPS exposure decreases the parthenogenetic development of bovine oocytes. (A) Rates of different stages of parthenogenetic development after oocytes were preincubated with LPS for $22 \mathrm{~h}$ during in vitro maturation. (B) Total number of cells in blastocysts from each treatment group. (C) Representative photomicrographs. Blue, chromatin; Cyan, the transferase-mediated dUTP nick end labeling (TUNEL)-positive apoptotic nuclei. Bar $=20 \mu \mathrm{m}$. LPS, lipopolysaccharide. (D) Apoptotic cell rate. Asterisk indicates significant difference $(p<0.05)$.

lation process. In the current study, exposure of COCs to LPS upregulated the TLR4 $\mathrm{mRNA}$ and protein levels, and increased the concentrations of IL- $1 \beta$, TNF- $\alpha$, and IL- 6 in the supernatant in the presence of LPS. Furthermore, p38 MAPK and NF- $\kappa B$ pathways were activated in response to LPS. We speculated that LPS could trigger inflammatory processes in COCs via the TLR4 pathway, and then affect oocyte maturation.

Previously, it has been demonstrated that the maturation and developmental competence of oocytes could be damaged in a bacterial-type-dependent manner [22]. Oocytes treated with gram-negative endotoxin (i.e., LPS) exhibited impaired nuclear and cytoplasmic maturation, characterized by a reduced proportion of MII oocytes and CGs distributed in the cortical region [23]. The number of oocytes that failed to reach the MII stage of meiosis or those with perturbed meiotic structures such as aberrant spindles, chromosomal ejection, and germinal vesicle breakdown failure was significantly increased following exposure to LPS [13]. Consistent with the above results, our study further confirmed that LPS exposure inhi- bited nuclear and cytoplasmic maturation of bovine oocytes. LPS is primarily recognized as a proinflammatory and immunomodulatory molecule [24]. However, many studies have demonstrated that LPS could release superoxide radicals and other ROS, leading to oxidative stress and programmed cell death in different cell lines $[14,25,26]$. As expected, LPS exposure significantly increased the intracellular ROS levels and significantly decreased the transcript abundance of detoxification enzymes including $\operatorname{Tr} x, \operatorname{Tr} x 2$, and $\operatorname{Pr} x 1$ in MII bovine oocytes. Additionally, LPS-challenged oocytes exhibited significant increases in the early apoptotic rate and released cytochrome $C$. In a recent study on human gingival fibroblasts, LPS was found to markedly elevate mitochondrial and cytoplasmic ROS levels [27]. Similarly, another study found elevated apoptotic cell rate and cytosolic cytochrome $C$ and caspase- 3 activities in rat alveolar epithelial cells in vivo and in vitro after challenge with LPS [28]. The similar results obtained in this study suggest that the detrimental effects of LPS on oocyte maturation potential may be partly via the in- 
duction of oxidative stress and apoptosis.

Given the prominent effect of LPS on oocyte maturation, we further evaluated the effect of LPS on the embryonic development and quality of LPS-treated parthenogenetically activated oocytes. The cleavage, morula, and blastocyst formation rates showed obvious decreases in the oocytes that had been exposed to LPS. LPS significantly increased the apoptotic signals but not the total number of nuclei in embryos. LPS has been associated with adverse developmental outcomes such as embryonic resorption, fetal death, and growth retardation [29]. In a previous study, oocytes that had been matured in follicular fluid aspirated from LPS-induced mastitic cows showed significantly impaired developmental competence [23]. Ibrahim et al [8] found that LPS could adversely affect the proportion of embryos that develop to the blastocyst stage. Our results are consistent with reports from previous literature and suggest that exposure of oocytes to environments exposed to LPS could reduce their developmental potential.

\section{CONCLUSION}

In conclusion, our findings demonstrate that LPS could reduce the maturation ability and parthenogenetic developmental competence of bovine oocytes in vitro. Our findings suggest that the immune response, oxidative stress, and pro-apoptotic effects of LPS may be the potential mechanisms underlying this effect.

\section{CONFLICT OF INTEREST}

We certify that there is no conflict of interest with any financial organization regarding the material discussed in the manuscript.

\section{ACKNOWLEDGMENTS}

This work was supported by the Agricultural Science and Technology Innovation Program (ASTIP-2016-IAS-06), the China Agriculture Research System (CARS-36) and the Chinese Academy of Agricultural Science and Technology Innovation Project (CAAS-XTCX2016011).

\section{REFERENCES}

1. Sheldon IM, Noakes DE, Rycroft AN, Pfeiffer DU, Dobson $\mathrm{H}$. Influence of uterine bacterial contamination after parturition on ovarian dominant follicle selection and follicle growth and function in cattle. Reproduction 2002;123:837-45.

2. Williams EJ, Sibley K, Miller AN, et al. The effect of Escherichia coli lipopolysaccharide and tumour necrosis factor alpha on ovarian function. Am J Reprod Immunol 2008;60:462-73.

3. Herath S, Williams EJ, Lilly ST, et al. Ovarian follicular cells have innate immune capabilities that modulate their endocrine function. Reproduction 2007;134:683-93.

4. Borsberry S, Dobson H. Periparturient diseases and their effect on reproductive performance in five dairy herds. Vet Rec 1989; 124:217-9.

5. LeBlanc SJ, Duffield TF, Leslie KE, et al. Defining and diagnosing postpartum clinical endometritis and its impact on reproductive performance in dairy cows. J Dairy Sci 2002;85:2223-36.

6. Karsch FJ, Battaglia DF, Breen KM, Debus N, Harris TG. Mechanisms for ovarian cycle disruption by immune/inflammatory stress. Stress 2002;5:101-12.

7. Sheldon IM, Cronin J, Goetze L, Donofrio G, Schuberth HJ. Defining postpartum uterine disease and the mechanisms of infection and immunity in the female reproductive tract in cattle. Biol Reprod 2009;81:1025-32.

8. Ibrahim S, Salilew-Wondim D, Rings F, et al. Expression pattern of inflammatory response genes and their regulatory micrornas in bovine oviductal cells in response to lipopolysaccharide: implication for early embryonic development. PLoS One 2015; 10:e0119388.

9. Raetz CR, Whitfield C. Lipopolysaccharide endotoxins. Annu Rev Biochem 2002;71:635-700.

10. Beutler B. Inferences, questions and possibilities in Toll-like receptor signalling. Nature 2004;430:257-63.

11. Doyle SL, O'Neill LA. Toll-like receptors: from the discovery of NFkappaB to new insights into transcriptional regulations in innate immunity. Biochem Pharmacol 2006;72:1102-13.

12. Blum JW, Dosogne H, Hoeben D, et al. Tumor necrosis factoralpha and nitrite/nitrate responses during acute mastitis induced by Escherichia coli infection and endotoxin in dairy cows. Domest Anim Endocrinol 2000;19:223-35.

13. Bromfield JJ, Sheldon IM. Lipopolysaccharide initiates inflammation in bovine granulosa cells via the TLR4 pathway and perturbs oocyte meiotic progression in vitro. Endocrinology 2011;152:5029-40.

14. Raza H, John A, Shafarin J. Potentiation of LPS-induced apoptotic cell death in human hepatoma HepG2 cells by aspirin via ROS and mitochondrial dysfunction: protection by $\mathrm{N}$-acetyl cysteine. PLoS One 2016;11:e0159750.

15. Coticchio G, Dal Canto M, Mignini Renzini M, et al. Oocyte maturation: gamete-somatic cells interactions, meiotic resumption, cytoskeletal dynamics and cytoplasmic reorganization. Hum Reprod Update 2015;21:427-54.

16. Coticchio G, Sereni E, Serrao L, Mazzone S, Iadarola I, Borini A. What criteria for the definition of oocyte quality? Ann NY Acad Sci 2004;1034:132-44.

17. Platz-Christensen JJ, Mattsby-Baltzer I, Thomsen P, Wiqvist N. Endotoxin and interleukin-1 alpha in the cervical mucus and vaginal fluid of pregnant women with bacterial vaginosis. Am J Obstet Gynecol 1993;169:1161-6.

18. Bannerman DD, Paape MJ, Hare WR, Sohn EJ. Increased levels of LPS-binding protein in bovine blood and milk following 
bacterial lipopolysaccharide challenge. J Dairy Sci 2003;86: 3128-37.

19. Thouas GA, Korfiatis NA, French AJ, Jones GM, Trounson AO. Simplified technique for differential staining of inner cell mass and trophectoderm cells of mouse and bovine blastocysts. Reprod Biomed Online 2001;3:25-9.

20. Shimada M, Hernandez-Gonzalez I, Gonzalez-Robanya I, Richards JS. Induced expression of pattern recognition receptors in cumulus oocyte complexes: novel evidence for innate immune-like functions during ovulation. Mol Endocrinol 2006; 20:3228-39.

21. Takeuchi O, Akira S. Pattern recognition receptors and inflammation. Cell 2010;140:805-20.

22. Roth Z, Asaf S, Furman O, et al. Subclinical mastitis disrupts oocyte cytoplasmic maturation in association with reduced developmental competence and impaired gene expression in preimplantation bovine embryos. Reprod Fertil Dev 2015 Apr 20 [Epub]. https://doi.org/10.1071/RD14431

23. Asaf S, Leitner G, Furman O, et al. Effects of Escherichia coliand Staphylococcus aureus-induced mastitis in lactating cows on oocyte developmental competence. Reproduction 2014;147:33-43.
24. Jaja-Chimedza A, Gantar M, Mayer GD, Gibbs PD, Berry JP. Effects of cyanobacterial lipopolysaccharides from microcystis on glutathione-based detoxification pathways in the zebrafish (Danio rerio) embryo. Toxins (Basel) 2012;4:390-404.

25. Sagar S, Kumar P, Behera RR, Pal A. Effects of CEES and LPS synergistically stimulate oxidative stress inactivates OGG1 signaling in macrophage cells. J Hazard Mater 2014;278:236-49.

26. Qin T, Yin Y, Yu Q, Yang Q. Bursopentin (BP5) protects dendritic cells from lipopolysaccharide-induced oxidative stress for immunosuppression. PLoS One 2015;10:e0117477.

27. Li X, Wang X, Zheng M, Luan QX. Mitochondrial reactive oxygen species mediate the lipopolysaccharide-induced proinflammatory response in human gingival fibroblasts. Exp Cell Res 2016;347:212-21.

28. Fu C, Dai X, Yang Y, Lin M, Cai Y, Cai S. Dexmedetomidine attenuates lipopolysaccharide-induced acute lung injury by inhibiting oxidative stress, mitochondrial dysfunction and apoptosis in rats. Mol Med Rep 2017;15:131-8.

29. Zhao L, Chen YH, Wang H, et al. Reactive oxygen species contribute to lipopolysaccharide-induced teratogenesis in mice. Toxicol Sci 2008;103:149-57. 